\title{
The Case of IgG4-related Ophthalmic Disease Accompanied by Compressive Optic Neuropathy
}

\author{
Marie Takeishi, Toshiyuki Oshitari, Satomi Ota, Takayuki Baba, and Shuichi Yamamoto \\ Department of Ophthalmology and Visual Science, Chiba University Graduate School of Medicine, Chiba, Japan
}

\begin{abstract}
A 76-year-old woman was diagnosed with thyroid-associated orbitopathy (TAO) associated with hypothyroidism at another clinic. Magnetic resonance imaging showed enlargements of the extraocular muscles. Because her best-corrected visual acuity (BCVA) was 0.010D, she was referred to the Chiba University Hospital. She had an elevation of serum IgG4. She was diagnosed with possible IgG4-related ophthalmic disease (IgG4-ROD) accompanied by optic nerve compression with the mass. She underwent methylprednisolone pulse therapy, and, two months later, her BCVA and visual field defect were greatly improved. Our findings indicate that ophthalmologists should be aware that IgG4-ROD can be masked by lesions associated with TAO.
\end{abstract}

\section{ARTICLE HISTORY}

Received 13 August 2017

Revised 25 September 2017

Accepted 4 October 2017

\section{KEYWORDS}

Compressive optic neuropathy; Hashimoto's thyroiditis; IgG4-related ophthalmic disease; methylprednisolone pulse therapy; thyroid-associated orbitopathy

\section{Introduction}

Immunoglobulin G4-related ophthalmic disease (IgG4-ROD) is a new clinical entity characterised by orbital inflammatory lesions or pseudotumour-like lesions with infiltration of IgG4-immunopositive plasmacytes and high serum IgG4 levels. ${ }^{1}$ The recently presented diagnostic criteria of IgG4-ROD indicated that histopathological examinations are required for a definitive diagnosis ${ }^{1}$ because other orbital diseases such as thyroid-associated orbitopathy must be considered in the differential diagnosis. ${ }^{1}$

Growing evidence has demonstrated a strong relationship between thyroid-associated orbitopathy and IgG4-ROD. ${ }^{2,3}$ Sy et al. reported that $8.3 \%$ of patients with thyroid-associated orbitopathy had high serum IgG4 levels of $\geq 135 \mathrm{mg} / \mathrm{dl}^{2}{ }^{2}$ The results of a recent study indicated that Hashimoto's thyroiditis can be divided into two subtypes based on those with and those without IgG4-positive plasma cell infiltration. ${ }^{3}$ Hashimoto's thyroiditis with IgG4-positive plasma cell infiltration is characterised by more rapid progression, a lower woman-to-man ratio, and higher circulating thyroid autoantibodies than those without IgG4-positive plasma cell infiltration. ${ }^{3}$

Very recently, Inaba et al. reported the first case of IgG4-ROD mimicking thyroid-associated orbitopathy in a patient with Hashimoto's thyroiditis. ${ }^{4}$ We report a second case of IgG4-ROD with Hashimoto's thyroiditis accompanied by compressive optic neuropathy.

\section{Case report}

A 76-year-old woman had been diagnosed with Hashimoto's thyroiditis with thyroid-associated orbitopathy and was followed at the Kimitsu Central Hospital from July 2015. Her right exophthalmos increased in October 2016, and a lagoophthalmic keratitis developed in February 2017. Magnetic resonance imaging (MRI) showed enlargements of several extraocular muscles, and blood tests showed high levels of serum IgG4 of 446 mg/dl. On 27 February 2017, her decimal bestcorrected visual acuity (BCVA) decreased markedly from 0.5 to $0.01 \mathrm{OD}$, and she was referred to the Chiba University Hospital.

Upon our initial examination, her decimal BCVA was counting finger OD and 0.7 OS. She had pain in her right eye and severe exophthalmos with lagoophthalmic keratitis (Figure 1). The critical flicker frequency (CFF) was $28.3 \mathrm{~Hz}$ OD and $27.3 \mathrm{~Hz}$ OS. The values of exophthalmos were $19 \mathrm{~mm}$ OD and $15 \mathrm{~mm}$ OS. Goldmann perimetry

CONTACT Toshiyuki Oshitari Tari@aol.com; oshitari@faculty.chiba-u.jp Department of Ophthalmology and Visual Science, Chiba University Graduate School of Medicine, Inohana 1-8-1, Chuo-ku, Chiba 260-8670, Japan.

Colour versions of one or more of the figures in the article can be found online at www.tandfonline.com/ioph.

๑) 2018 Taylor \& Francis 

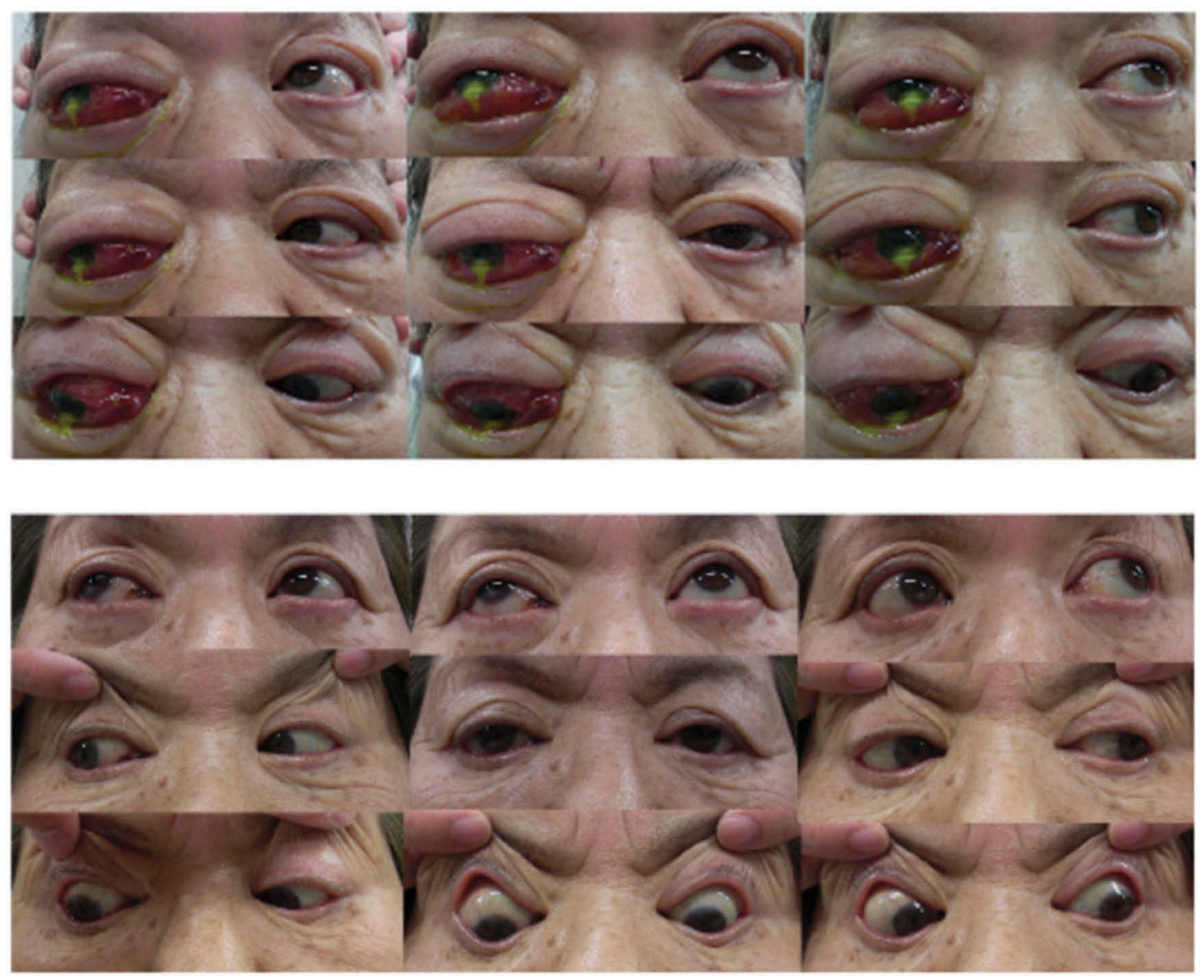

Figure 1. Ocular findings of a patient with thyroid ophthalmopathy before and after methylprednisolone pulse therapy. Marked exophthalmos with severe chemosis and lagoophthalmic keratitis is present in the right eye (upper panels). Ocular movements in the right upper field are severely limited (upper panels). After methylprednisolone pulse therapy, the severe exophthalmos was significantly improved (lower panels). No clinical symptoms including ocular movement restrictions were found after the methylprednisolone pulse therapy (lower panels). Lagoophthalmic keratitis was also improved (lower panels).

showed a lower right visual field defect, and Humphry visual field examinations showed right lower visual field defects, bilateral peripheral visual field defects and right central scotoma (Figure 2). The ocular movements in the right upper field were severely limited because of a mass that occupied the upper orbit (Figure 3). MRI showed multiple extracellular muscles enlargements and a large mass that occupied the upper orbit in both eyes (Figure 4). The mass may be derived from the lacrimal glands. Right optic nerve was compressed with the mass. But, left optic nerve was also compressed with the mass mildly. Blood tests showed that the level of interleukin-2R was $377 \mathrm{U} / \mathrm{ml}$, C-reactive protein was $0.04 \mathrm{mg} / \mathrm{dl}$ and thyroid stimulating hormone was $21.4 \mu \mathrm{l} / \mathrm{U} / \mathrm{ml}$. She was diagnosed with possible IgG4-ROD accompanied by compressive optic neuropathy. Because the mass was too deep in the orbit and difficult to perform biopsy and was causing severe compressive optic neuropathy, two cycles of methylprednisolone pulse therapy with intravenous antibiotics was performed immediately without histopathological examinations. Two months later, her visual acuity improved to $0.4 \mathrm{OD}$, and the visual field defects and ocular movements were markedly improved (Figures 2 and 3). MRI showed a significant reduction in the size of the mass in the upper orbit (Figure 4). The lagoophthalmic keratitis also improved because the exophthalmos was reduced to $14 \mathrm{~mm}$ OD and $14 \mathrm{~mm}$ OS (Figure 1). Oral predonisolone was tapered to $20 \mathrm{mg} /$ day, and no side effects of the steroid therapy were observed. Four months after the methylprednisolone pulse therapy, the final BCVAs were 0.6 OD and 0.6 OS, and the CFFs were $35.0 \mathrm{~Hz}$ OD and $34.2 \mathrm{~Hz}$ OS. No double vision was present. 

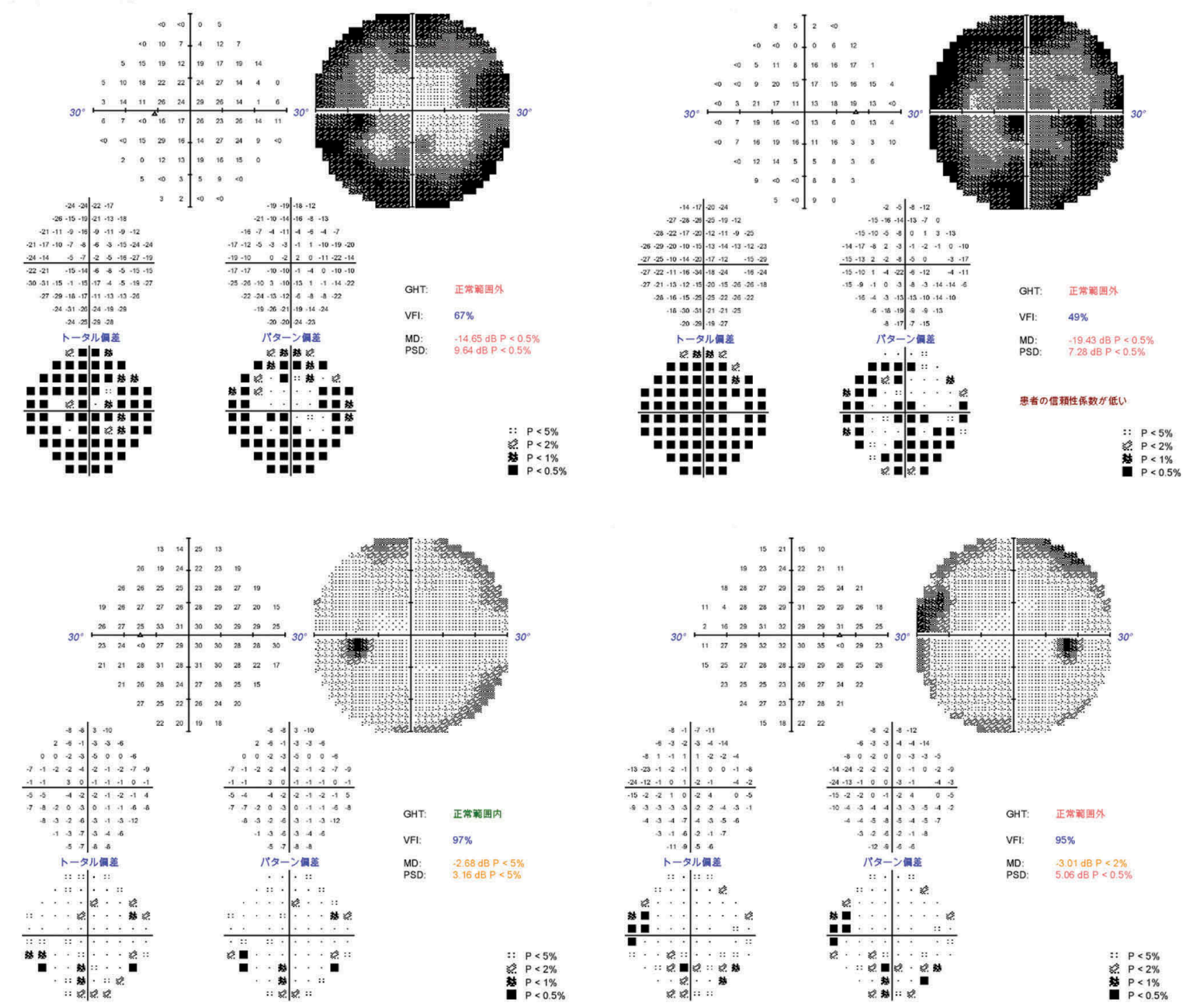

Figure 2. Humphry visual field analysis before (upper panels) and after methylprednisolone pulse therapy (lower panels). Before methylprednisolone pulse therapy, peripheral visual field defects were found in the both eyes (upper panels). A central scotoma was found in the right eye (right upper panel). Two months after methylprednisolone pulse therapy, the visual field defects are significantly improved in both eyes (lower panels).

\section{Discussion}

The presence of multiple masses in the orbit and elevated serum IgG4 are diagnostic criteria for 'possible' IgG4-ROD. ' Although histopathological examinations were not performed in this case, the marked improvement after steroid therapy indicated that the mass in the upper orbit was most likely due to IgG4-ROD.

Thyroid-associated orbitopathy occurs in a low percentage of patients with Hashimoto's thyroiditis. Thus, IgG4-ROD can be masked by thyroid-associated orbitopathy in some cases. ${ }^{4}$ Inaba et al. reported the first case of IgG4-ROD with accompanying Hashimoto's thyroiditis. ${ }^{4}$ Their patient had been treated with inhaled corticosteroids for bronchial asthma for 9 years. ${ }^{4}$ Although their patient had multiple lesions in his body, the progression was slow. ${ }^{4}$ Thus, no aggressive treatment was performed, and IgG4ROD spontaneously improved. ${ }^{4}$ However, it is known that IgG4-positive Hashimoto's thyroiditis has a more rapid progression than IgG4-negative Hashimoto's thyroiditis. ${ }^{3}$ It is not known whether IgG4-ROD accompanied by Hashimoto's thyroiditis progresses rapidly or not. However, Bozkirli et al. reported that IgG4 may be responsible for the increase in the 

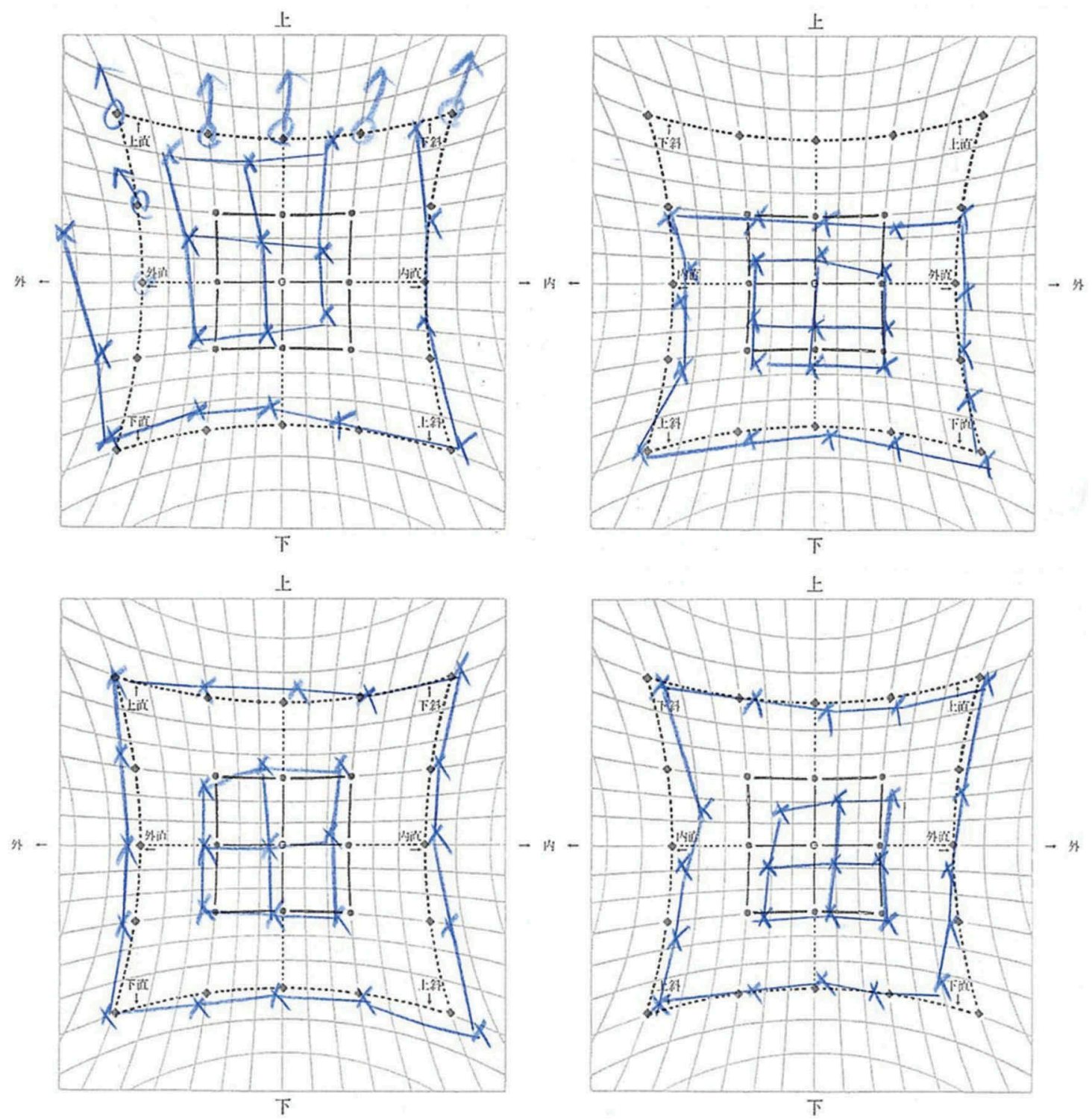

Figure 3. HESS charts before (upper panels) and after (lower panels) methylprednisolone pulse therapy. Before the methylprednisolone pulse therapy, the ocular movements to the right upper field were severely limited (upper panels). Two months after methylprednisolone pulse therapy, ocular movement was significantly improved. No diplopia was noticed after the therapy.

production of TSH-receptor antibodies in plasma cells in the orbital tissue, and that the elevation of serum IgG4 levels may be an early indicator of acute inflammation of thyroid-associated orbitopathy. ${ }^{5}$ Thus, when thyroid-associated orbitopathy is markedly exacerbated, ophthalmologists should consider IgG4-ROD that can be masked by lesions associated with thyroid-associated orbitopathy.

A late diagnosis of IgG4-ROD can lead to permanent optic nerve damage because a large mass lesion can compress the optic nerve at the late stage of IgG4-ROD. Takahashi et al. reported a case of bilateral compressive optic neuropathy accompanied by
IgG4-ROD. ${ }^{6}$ Similar to our case, both the right and left optic nerves were mildly compressed. Because optic nerve compression can lead to permanent loss of vision, early diagnosis is needed to begin appropriate treatment.

In conclusion, ophthalmologists should examine serum IgG4 levels when thyroid-associated orbitopathy is markedly exacerbated. This is because IgG4-ROD may have pathological links with thyroid-associated orbitopathy ${ }^{5}$ and because IgG4-ROD can be masked by lesions associated with thyroid-associated orbitopathy. 

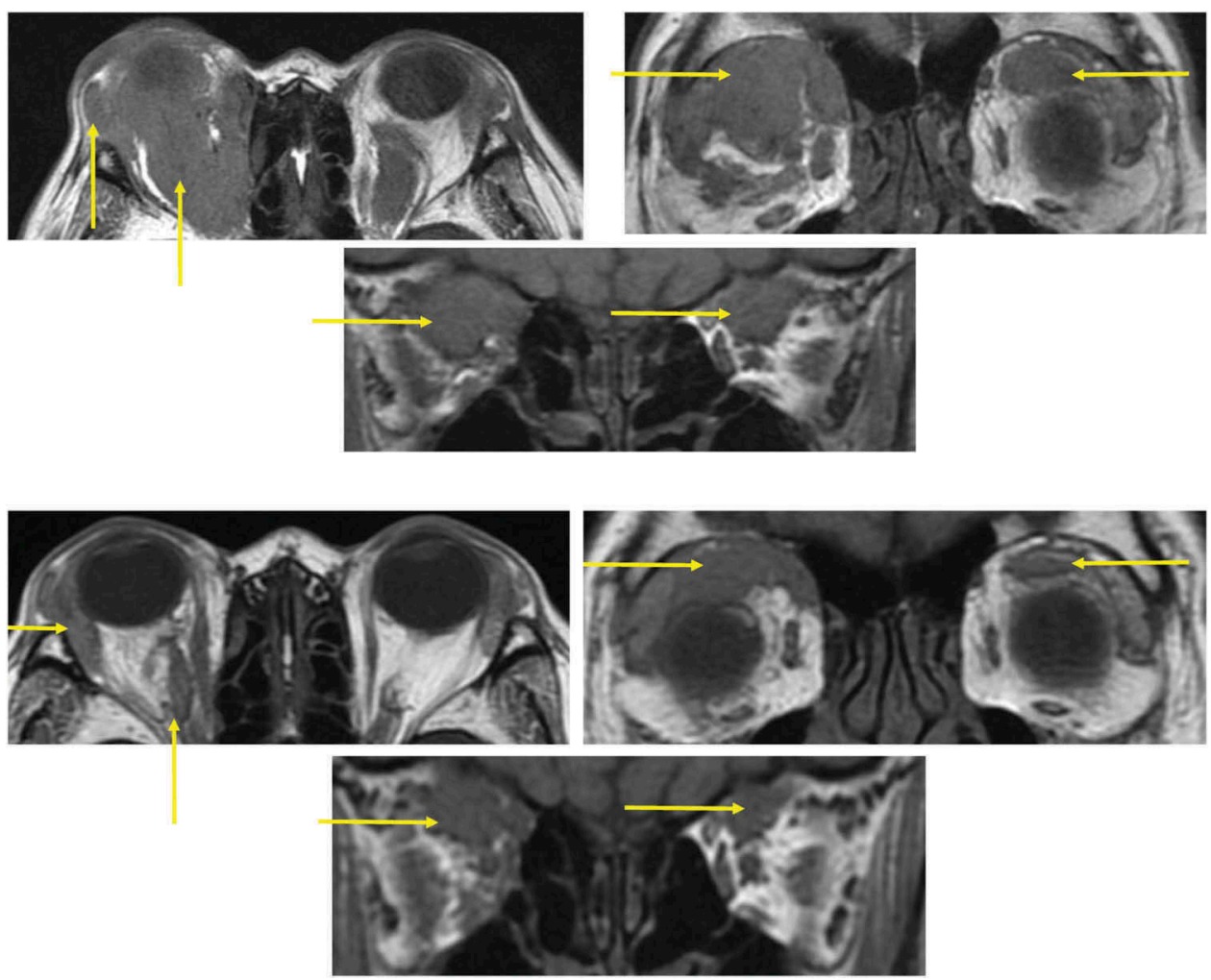

Figure 4. MRI findings before (upper panels) and after (lower panels) steroid pulse therapy. Before methylprednisolone pulse therapy, larger mass lesions (yellow arrows) are present in the upper orbital cavity in the right (upper panels). Right optic nerve was compressed with the mass and left optic nerve was also compressed with the mass mildly (upper panels). Multiple extraocular muscles were also enlarged (upper panels). Two months after methylprednisolone pulse therapy, the mass lesion was significantly reduced (lower panels).

\section{Acknowledgements}

The authors thank Professor Emeritus Duco Hamasaki of the Bascom Palmer Eye Institute of the University of Miami, FL for editing the manuscript.

\section{Declaration of interest}

The authors report no conflicts of interest. The authors alone are responsible for the content and writing of the paper.

\section{Funding}

This work was supported by a Grant-in-Aid from the Ministry of Education, Science, Sports and Culture of the Japanese Government (16K11318).

\section{References}

1. Goto H, Takahira M, Azumi A. Japanese study group for IgG4-related ophthalmic disease. Diagnostic criteria for IgG4-related ophthalmic disease. Jpn J Ophthalmol 2015;59:1-7. doi:10.1007/s10384-014-0352-2.
2. Sy A, Silkiss RZ. Serum total IgG and IgG4 levels in thyroid eye disease. Int Med Case Rep J 2016;9:325328. doi:10.2147/IMCRJ.S116331.

3. Li Y, Nishihara E, Hirokawa M, Taniguchi E, Miyauchi A, Kakudo K. Distinct clinical, serological, and sonographic characteristics of hashimoto's thyroiditis based with and without IgG4-positive plasma cells. J Clin Endocrinol Metab 2010;95:1309-1317. doi:10.1210/jc.2009-1794.

4. Inaba $\mathrm{H}$, Hayakawa $\mathrm{T}$, Miyamoto $\mathrm{W}$, Takeshima $\mathrm{K}$, Yamaoka H, Furukawa Y, Kawashima H, Ariyasu H, Wakasaki H, Furuta H, Nishi M, Nakao T, Sasaki H, Okada Y, Matsunaga K, Nakamura Y, Akamizu T. IgG4-related ocular adnexal disease mimicking thyroid-associated orbitopathy. Intern Med 2013;52:25452551. doi:10.2169/internalmedicine.52.0902.

5. Bozkirli E, Bakiner OS, Ersozlu Bozkirli ED, Eksi Haydardedeoglu F, Sizmaz S, Torun AI, Ertorer ME. Serum immunoglobulin G4 levels are elevated in patients with Graves' ophthalmopathy. Clin Endocrinol (Oxf) 2015;83:962-967. doi:10.1111/cen.12671.

6. Takahashi Y, Kitamura A, Kakizaki H. Bilateral optic nerve involvement in immunoglobulin G4-related ophthalmic disease. J Neuroophthalmol 2014;34:16-19. doi:10.1097/WNO.0b013e3182a304f4. 